\title{
Le vocabulaire des établissements urbains antiques et les incertitudes du mot castellum en Afrique
}

The Vocabulary of the Ancient Urban Settlements and the Uncertainties of the Word Castellum in Africa

\section{Anne-Florence Baroni}

\section{(2) OpenEdition}

Journals

Édition électronique

URL : https://journals.openedition.org/gaia/792

DOI : $10.4000 /$ gaia.792

ISSN : 2275-4776

Éditeur

UGA Éditions/Université Grenoble Alpes

Édition imprimée

ISBN : 978-2-37747-199-7

ISSN : 1287-3349

\section{Référence électronique}

Anne-Florence Baroni, « Le vocabulaire des établissements urbains antiques et les incertitudes du mot castellum en Afrique », Gaia [En ligne], 22-23 | 2020, mis en ligne le 01 juin 2020, consulté le 28 juin 2022. URL : http://journals.openedition.org/gaia/792 ; DOI : https://doi.org/10.4000/gaia.792

Ce document a été généré automatiquement le 9 décembre 2021.

Gaia. Revue interdisciplinaire sur la Grèce archaïque 


\section{Le vocabulaire des établissements urbains antiques et les incertitudes du mot castellum en Afrique ${ }^{1}$}

The Vocabulary of the Ancient Urban Settlements and the Uncertainties of the Word Castellum in Africa

Anne-Florence Baroni

1 À l'époque tardo-républicaine, une formule récurrente de la lex de Gallia Cisalpina donne les noms des différents lieux où les dispositions de cette loi s'appliquent: oppidum, municipium, colonia, praefectura, forum, uicus, conciliabulum, castellum, territorium ${ }^{2}$. Cette liste illustre à la fois la diversité, la complexité et l'ambiguïté pour l'historien des termes latins employés pour désigner les catégories d'habitat: tandis que les trois premiers sont généralement considérés comme des villes, le caractère urbain est plus difficile à déterminer pour les autres. On sait par ailleurs que l'éventail de ces termes, forgés en Italie pour caractériser les formes urbaines de la péninsule, ne se trouve pas intégralement ni de manière uniforme dans la documentation des provinces romaines. L'écart entre la liste de la lex de Gallia Cisalpina et les attestations du vocabulaire désignant les établissements urbains dans les provinces pose donc à la fois la question de la définition de la ville pour les Romains, notamment face aux réalités institutionnelles et urbaines dans les territoires conquis, et celle de l'adaptation des noms latins pour désigner ces réalités. Particulièrement dans les régions où l'organisation en cité n'existe pas avant l'occupation romaine, l'étude du vocabulaire de la ville invite non seulement à s'interroger sur la catégorisation romaine de ce que les conquérants ont trouvé sur place, mais aussi sur la polysémie éventuelle des termes employés. Si tant est qu'elle ne soit pas simplement due à notre mauvaise compréhension des sources, la polysémie apparente peut cacher une adaptation aux réalités locales, mais aussi une évolution institutionnelle, urbaine ou chronologique.

2 L'une des spécificités de la documentation des provinces africaines est le nombre important de castella, qui s'oppose à la rareté des uici et à l'absence des fora, pourtant fréquents au nord de la Méditerranée. Le terme fait partie des mots désignant une 
agglomération dont on peine à saisir le sens exact, aussi bien en ce qui concerne la forme urbaine que le statut juridique éventuellement précisés par ce terme ${ }^{3}$. En l'état actuel de la bibliographie, la notice de René Rebuffat dans l'Encyclopédie berbère ${ }^{4}$ demeure la seule tentative de synthèse consacrée spécifiquement au castellum dans les provinces africaines : tout en refusant de se prononcer sur une définition précise du terme, R. Rebuffat a recensé une soixantaine de castella, principalement à l'ouest de la Proconsulaire (dans les limites des $\mathrm{I}^{\mathrm{er}}$ et $\mathrm{II}^{\mathrm{e}}$ siècles, qui incluent la Numidie) et en Maurétanie césarienne et sitifienne. Une étude approfondie du castellum et une réflexion semblable à celle dont a par exemple bénéficié le uicus en Europe reste donc à mener $^{5}$; il conviendrait également de comparer la situation africaine à celle des autres provinces. Le but de cet article n'est donc pas de s'attacher à l'ensemble des problèmes posés par le castellum, mais de proposer une brève présentation des définitions les plus courantes dans la bibliographie. Celles-ci mettent principalement en valeur la question de la fortification du castellum, de son rapport avec le pagus, et de son statut pérégrin ${ }^{6}$. On verra qu'aucun de ces éléments de définition n'est complètement assuré.

\section{Une agglomération fortifiée?}

3 Les Étymologies d'Isidore de Séville offrent une aide limitée pour définir le vocabulaire de la ville, car l'auteur du $\mathrm{vI}^{\mathrm{e}}-\mathrm{VII}^{\mathrm{e}} \mathrm{s}$. y décrit une réalité depuis longtemps disparue ${ }^{7}$. Synthétiques et peu précises, parfois contradictoires, elles sont naturellement sujettes à caution et ne sont pas toujours vérifiables dans la documentation, quand celle-ci est disponible. Toutefois, en l'absence de certitudes sur la signification exacte du terme, elles ne peuvent être exclues a priori de la réflexion sur le castellum.

Isidore de Séville rappelle l'étymologie du mot (Etymologiae, XV, II, 13) ${ }^{8}$ : Castrum antiqui dicebant oppidum loco altissimo situm, quasi casa altam; cuius pluralis numerus castra, diminutiuum castellum est; siue quod castrabatur licencia inibi habitantium, ne passim uaga hosti pateret. [" Castrum était le nom donné par les anciens à un oppidum situé en un endroit élevé : c'est comme une maison haute; le pluriel de ce mot est castra, son diminutif castellum ; ou bien ce mot vient de ce qu'y était restreinte la liberté de ceux qui y habitaient, pour éviter que leur dispersion n'ouvrît un accès à l'ennemi ${ }^{9}$. »] Cette étymologie explique le nom de castellum donné à des forts militaires - par exemple, pour l'Afrique, castellum Dimmidi et Tamuda ${ }^{10}$ - sur lesquels nous ne reviendrons pas ici. L'idée de fortification a également été retenue pour les agglomérations «civiles " qui, comme dans la lex de Gallia Cisalpina, sont appelées castellum ${ }^{11}$. Pour ces dernières, la traduction par «bourg fortifié " ou "agglomération fortifiée » est couramment adoptée $^{12}$. La forte proportion de récits de guerre dans les sources littéraires qui mentionnent des castella en Afrique et le fait que castellum soit également employé pour désigner des installations militaires permet en effet d'imaginer que ces agglomérations bénéficient, si ce n'est d'un rempart, du moins d'une position forte. C'est pourquoi Jehan Desanges a pu définir le castellum comme « une agglomération indigène fortifiée par l'art ou par l'assise ${ }^{13}$ ».

5 Cette solution prudente est toutefois infirmée par un passage de Justin (XXII, 5, 5). Dans le récit de l'expédition d'Agathoclès, il est ainsi précisé qu'en Afrique, les castella ne sont pas nécessairement fortifiés: Huc accedere, quod urbes castellaque Africae non muris cinctae, non in montibus positae sint, sed in planis campis sine ullis munimentis iaceant [...]. [«À cela s'ajoute que les villes et les castella de l'Afrique ne sont ni entourés de 
murailles, ni construits sur des montagnes, mais sont situés dans les plaines sans être aucunement fortifiés ${ }^{14}$. $\left.)\right]$

6 Si un mur d'enceinte est bien attesté pour certains castella ${ }^{15}$, son absence est également explicite dans plusieurs sources épigraphiques, notamment une série d'inscriptions maurétaniennes gravées sous le règne de Sévère Alexandre: cinq textes semblables signalent la construction de murs dans les castella au sud de Sétif, en 227 au castellum Citofactense (Kherbet Aïn Soltane) ${ }^{16}$, à Perdices (Kherbet el Marder) ${ }^{17}$ et à Aïn el Hadjar ${ }^{18}$, puis en 234 au castellum Dianense (Gellal, près d'Aïn Melloul) ${ }^{19}$, et à une date inconnue au castellum Thib(---) (Aïn Melloul) ${ }^{20}$. Comme l'a noté Jérôme Carcopino, l'éditeur de l'inscription de Kherbet Aïn Soltane, le castellum préexiste à la construction d'une muraille car "c'est seulement quand le castellum eut vu grandir son importance, et s'accroître ses bâtiments intérieurs - auctis uiribus et moenibus suis - que les castellani élevèrent ses remparts - muros extruxerunt $[. . .]^{21}$ ». De même, en 243 sous Gordien III, les colons d'un domaine impérial érigent une muraille au castellum Cellense (Kherbet Zerga $^{22}$.

7 L'absence de murs est en outre retenue par Isidore de Séville, bien qu'il ait d'abord rappelé que castellum était un diminutif de castrum. Selon lui, l'oppidum dispose de murailles, mais le castellum, comme le uicus et le pagus, n'en a pas (Etymologiae, XV, II, 6) : oppidum autem magnitudine et moenibus discrepare a uico et castello et pago [" l'oppidum diffère du uicus, du castellum et du pagus par sa taille et par ses murailles »]. Ne reste ensuite dans les définitions d'Isidore que l'idée de petit centre urbain, sans statut juridique particulier :

- XV, II, 7 : ciuitates autem aut coloniae aut municipia aut uici aut castella aut pagi appellantur [« les cités sont appelées colonies, municipes, uici, castella ou pagi $\left.{ }^{23} »\right]$;

- XV, II, 11 : uici et castella et pagi hi sunt qui nulla dignitate ciuitatis ornantur, sed uulgari hominum conuentu incoluntur et propter paruitatem sui maioribus ciuitatibus adtribuuntur [« les uici, les castella et les pagi sont [des localités] qui ne sont en rien pourvues de la dignité d'une cité, mais sont habitées par un simple groupement d'hommes, et en raison de leur petite taille, sont attribuées à des cités plus grandes $\left.{ }^{24} »\right]$.

8 La contradiction entre les deux passages montre bien les difficultés d'Isidore pour synthétiser les informations de sa documentation: sont d'abord considérées comme ciuitates aussi bien les colonies et les municipes que les uici, castella et pagi (Etymologiae, XV, II, 7) ; puis, les uici, castella et pagi sont distingués de la ciuitas puisqu'ils n'en ont pas la dignité (Etymologiae, XV, II, 11). La contradiction peut se résoudre si on estime que ciuitas est un terme polysémique, comme notre terme " cité ", d'abord employé au sens de «ville» ou d'" agglomération » dans le premier passage, et au sens juridique dans le second ; mais elle rappelle combien l'utilisation des Étymologies est délicate.

\section{Pagus et castellum}

9 La formule d'Isidore aut coloniae aut municipia aut uici aut castella aut pagi explique peutêtre que, dans la bibliographie ancienne, le pagus ait parfois été mis au nombre des termes servant à désigner une agglomération et qu'il ait existé des confusions entre pagus et castellum ${ }^{25}$. Cependant, dans ses écrits sur les dépendances de la Confédération cirtéenne ${ }^{26}$, Stéphane Gsell fait déjà clairement la distinction entre les deux ${ }^{27}$ : les pagi sont des "districts ruraux dépendants de Cirta», tandis que les castella sont les agglomérations qui «s'élevaient » sur les pagi. Le pagus a fait l'objet ces vingt dernières 
années d'une série d'études qui ont confirmé que le pagus est avant tout d'une circonscription territoriale ${ }^{28}$. Le castellum n'a, en revanche, pas bénéficié de la même attention ; les définitions que l'on trouve dans la bibliographie reprennent plus souvent les interprétations des prédécesseurs qu'elles ne s'appuient sur une étude de la documentation. Le risque est alors de voir admettre des définitions inexactes, lorsque disparaît progressivement la référence au premier rédacteur et que les modifications successives du vocabulaire employé entraînent un changement de sens. Ainsi, à partir de l'idée de S. Gsell que le castellum est une agglomération établie sur la portion du territoire colonial qu'est le pagus, est apparue par glissement sémantique la définition de castellum comme "chef-lieu du pagus». Cette définition est par exemple le premier sens du mot castellum retenu par Samir Aounallah dans son ouvrage Pagus, castellum et ciuitas $^{29}$. Or, le "chef-lieu» désigne le centre administratif d'une circonscription territoriale ; il ne peut donc y avoir qu'un « chef-lieu » par circonscription. L'objection majeure à l'interprétation qui fait du castellum le chef-lieu du pagus est la difficulté, voire l'impossibilité, dans l'état actuel des recherches, à prouver qu'il existe un seul castellum par pagus. Certains castella peu éloignés les uns des autres ont pu faire partie d'une même circonscription territoriale. C'est le cas par exemple, en Numidie, des castella Elefant(...), Mastarense, Phuensium, Arsacalitanum, à l'ouest du territoire de Cirta ${ }^{30}$, où les attestations du pagus sont relativement rares par rapport au nombre de castella ${ }^{31}$. Deux autres dépendances de Cirta, le castellum Cald(is) ${ }^{32}$ et le castellum [---]alla, qui n'est pas autrement connu, sont associés sur une même inscription ${ }^{33}$ : peut-être disposaientils d'un lieu de représentation commun. Aucune des deux solutions (un seul castellum ou, au contraire, plusieurs castella par pagus) ne peut être prouvée. De plus, dans certains pagi, comme Thibilis, le castellum n'est pas attesté. La définition du castellum comme "chef-lieu» du pagus présente une autre difficulté: le pagus dispose-t-il juridiquement d'un "chef-lieu », c'est-à-dire d'un centre administratif défini ? Comme le notait déjà S. Gsell ${ }^{34}$, un lieu doit être prévu dans le pagus pour les archives et les activités des autorités du pagus. Néanmoins, il n'est pas sûr que l'agglomération où se tiennent ces activités soit en droit un «chef-lieu». On laissera la réponse aux spécialistes du pagus; mais, d'après les travaux récents menés sur l'Italie et les provinces occidentales, le pagus est une unité territorialement définie et une circonscription censitaire et fiscale, non pourvue d'un centre déterminé ${ }^{35}$. Il n'est donc pas établi que le castellum puisse être considéré comme le chef-lieu du pagus.

\section{Une agglomération indigène de statut pérégrin ?}

10 Le troisième élément de définition fréquent dans la bibliographie fait du castellum, selon la formule d'Azzedine Beschaouch largement reprise, "une agglomération indigène de statut pérégrin ${ }^{36}$ ". Les deux termes "indigène " et "pérégrin » sont à discuter. La fondation indigène des castella se vérifie dans bon nombre de cas, mais elle n'est pas systématique. Cela ressort nettement des remarques de R. Rebuffat dans la notice de l'Encyclopédie berbère. Certes, la majorité des toponymes des castella sont africains ; le toponyme, et souvent l'agglomération elle-même, existaient probablement avant la conquête : dans la plupart des cas, on peut donc dire, comme on le lit souvent, que le castellum est "d'origine indigène ». Cette " origine indigène » ne conditionne cependant pas le statut juridique de la communauté qui réside dans le castellum, ni de celui de ses habitants - la plupart des colonies en Afrique sont également d'anciennes agglomérations pré-romaines. Par ailleurs, certains castella ont des noms latins qui 
indiquent une création romaine ${ }^{37}$; ainsi, en Maurétanie, dans la région de Sétif, le castellum Aurelianense Antoninianense est fondé par les colons d'un domaine impérial en $213^{38}$. Castellum ne désigne donc pas uniquement des agglomérations antérieures à la conquête.

11 Il n'est pas sûr non plus que tous les castella soient des communautés pérégrines. On trouve la définition du castellum comme agglomération de statut pérégrin dans l'ouvrage fondamental de Claude Lepelley sur Les cités de l'Afrique romaine au BasEmpire $^{39}$; mais son succès semble dû essentiellement aux travaux d'Azzedine Beschaouch, notamment les articles sur le territoire de Sicca Veneria et de Cirta et sur l'histoire municipale d'Uchi Maius ${ }^{40}$. L'équivalence entre castellum et statut pérégrin est fréquente dans la bibliographie récente, au point que l'on tend à appeler castellum toute agglomération qui dispose d'institutions pérégrines, même quand le terme n'y est pas attesté. C'est le cas par exemple pour Chiniaua ${ }^{41}$ ou, dans la région d'Ammaedara, pour Thala ${ }^{42}$ et pour Tituli ${ }^{43}$, où la mention des seniores est tout particulièrement considérée comme l'indice de la présence d'un castellum ${ }^{44}$. Certes, les seniores kastelli sont attestés dans un castellum inconnu à Nibber ${ }^{45}$, à $U c u b i^{46}$ et à Aubuzza selon une inscription inédite ${ }^{47}$, c'est-à-dire dans les trois cas sur le territoire de Sicca Veneria ${ }^{48}$. Cependant, tous les castella ne sont pas administrés par des seniores ${ }^{49}$ et les attestations claires d'institutions pérégrines ne concernent qu'un petit nombre de castella. Il semble donc imprudent d'en tirer une conclusion générale.

12 La définition proposée par A. Beschaouch du castellum comme agglomération de statut pérégrin est pour sa part principalement fondée sur la comparaison qu'il a établie entre la documentation du territoire de Sicca Veneria, en Proconsulaire, et de Cirta, en Numidie $^{50}$, deux perticae dans lesquelles les inscriptions mentionnent des pagi et des castella. Pour A. Beschaouch, à Sicca, les pagi sont « des districts urbains de la colonie ", dont les citoyens sont « administrés par les duumvirs » tandis que les castella sont « des habitats pérégrins [...] où la population, restée foncièrement numide, était gouvernée par des conseils de notables âgés, sorte d'assemblées des anciens que les inscriptions, sous l'Empire, appellent seniores"; "le témoignage le plus éclatant de ces deux communautés » est l'inscription dédiée à Caracalla par les decur(iones) Sic(censes) Ucubi morantes et seniores $k$ (astelli) Ucubis ${ }^{51}$ : les decuriones Ucubi morantes seraient les représentants du pagus de citoyens romains d'Ucubi et les seniores $k$ (astelli) Ucubis, leur équivalent pour le castellum pérégrin de la même localité. Le castellum serait donc sur le territoire de Sicca l'équivalent de la ciuitas pérégrine de Thugga, dans la pertica carthaginoise, qui coexiste avec le pagus de citoyens romains ${ }^{52}$. La " coexistence de pagi romains et de castella numides » se vérifierait également dans les dépendances de Cirta. L'auteur en veut pour preuve les exemples de *Phua (castellum Phuensium), Sigus, et Celtianis (c'est-à-dire les trois localités de la pertica de Cirta où sont attestés à la fois un pagus et un castellum), ainsi que le castellum Tidditanorum où « un citoyen romain comme Q. Sittius C. fil. Quir. Urbanus ${ }^{53}$, qui a construit un temple de la Fortune Auguste et l'a doté d'une statue et d'un mobilier cultuel, n'a jamais pu être édile et questeur dans le castellum. De même, ce castellum ne pouvait être la patrie du préfet de Rome sous Antonin le Pieux, Q. Lollius Urbicus ».

13 La plupart des arguments de A. Beschaouch, pour Sicca Veneria comme pour Cirta, peuvent être réfutés. Dans le premier cas, le texte de la dédicace à Caracalla ne dit pas qu'existent à Ucubi à la fois un ordre de décurions et une assemblée de seniores: les decuriones Sicc(enses) prennent bien soin de préciser la cité dont ils sont membres du 
conseil, c'est-à-dire Sicca, et l'expression Ucubi morantes signifie simplement qu'ils résident à Ucubi ; ils ne sont donc pas les décurions d'un pagus de Sicca, comme l'entend A. Beschaouch, mais des décurions de la colonie de Sicca qui demeurent à Ucubi ${ }^{54}$; il n'existe donc pas de preuve de la coexistence d'un pagus de citoyens romains et d'une communauté pérégrine à Ucubi.

De plus, les témoignages cirtéens cités par A. Beschaouch peuvent également être interprétés d'une autre manière. La carrière de $Q$. Sittius $C$. fil. Quir. Urbanus n'est pas un argument déterminant en faveur de l'existence de deux communautés. Lorsque les seules fonctions connues pour un individu dans une dépendance de Cirta sont la questure et l'édilité, il est très difficile de déterminer si ces fonctions sont exercées dans la communauté locale (ici le castellum Tidditanorum ${ }^{55}$ ) ou au niveau de l'administration des quatre colonies cirtéennes: les agglomérations du territoire de Cirta disposent en effet d'institutions propres, notamment des décurions et un trésor public, depuis l'époque d'Hadrien au moins ${ }^{56}$; d'un autre côté, les inscriptions qui nous font connaître des magistrats de la Confédération cirtéenne ne proviennent pas uniquement de Cirta et sont disséminées sur l'ensemble du territoire, où les notables avaient des propriétés. Leur présence dans un castellum ne peut donc être utilisée comme argument pour déterminer le statut de la communauté locale. Quant au préfet de la Ville d'Antonin Q. Lollius Urbicus, on le considère comme originaire du castellum Tidditanorum, car il fait ériger son mausolée familial sur un domaine qu'il possède à proximités ${ }^{57}$. Cette propriété ne préjuge en rien du statut collectif du castellum voisin.

Aucune source n'atteste la présence d'institutions pérégrines ou d'un pagus au castellum Tidditanorum. En revanche, les auteurs d'un hommage à Q. Sittius Faustus, magistrat municipal à la fin du II $^{\mathrm{e}}$ siècle $^{58}$, se présentent comme les municipes, les concitoyens, de la personne qu'ils honorent ${ }^{59}$. Grâce à un passage célèbre d'Aulu-Gelle, on sait que le terme municipes désigne les citoyens d'un municipe ou d'une colonie ${ }^{60}$; il ne s'explique donc ici que parce que les «concitoyens» de Q. Sittius Faustus présents au castellum Tidditanorum sont des citoyens de la colonie romaine de Cirta, sur le territoire de laquelle se situe le castellum ${ }^{61}$. Pourtant, comme d'autres dépendances de Cirta à la même époque, la communauté dispose déjà d'institutions propres, en l'occurrence des décurions, qui autorisent l'érection du monument sur la place publique ${ }^{62}$. Or, à la différence de l'inscription d'Ucubi où l'on a pris soin de distinguer les decur(iones) Sic(censes) Ucubi morantes et les seniores k(astelli) Ucubis, les rédacteurs de l'hommage à Q. Sittius Faustus n'ont pas fait apparaître de différence entre la communauté des citoyens de la colonie et celle du castellum. Ce témoignage irait plutôt dans le sens non pas de la coexistence d'un pagus de citoyens romains et d'un castellum pérégrin, mais d'une communauté unique.

Surtout, comme l'avait déjà souligné Jacques Gascou dans son article consacré aux pagi et castella de la Confédération cirtéenne ${ }^{63}$, aucune inscription de Celtianis, de Sigus et du castellum Phuensium, où sont attestés à la fois pagus et castellum, ne permet d'établir une distinction juridique entre les deux termes. Au contraire, les inscriptions de la grotte Ez Zemma, dans le massif du Chettaba, à l'ouest de Constantine, laissent entendre que la différence entre le pagus et le castellum n'était pas extrêmement nette, au quotidien, dans l'esprit des habitants et de leurs représentants. Sur les parois de la grotte, des inscriptions sont gravées par les autorités du castellum Phuensium qui s'y rendent chaque année pour honorer une divinité dont le nom ne nous est parvenu que sous la forme des initiales G. D. A. ${ }^{64}$ : les magistrats des Phuenses sont nommés indifféremment 
pagi, magister Phuensium et, beaucoup plus rarement, magister kastelli ${ }^{65}$, alors qu'une seule communauté est attestée, celle des Phuenses. Il n'existe donc sur ce site aucune trace de double communauté, l'une de citoyens romains, l'autre de pérégrins. Les inscriptions du territoire de la Confédération cirtéenne ne confirment par conséquent aucunement la définition du castellum comme communauté de statut pérégrin.

Il est donc nécessaire de réserver le nom de castellum aux localités pour lequel le terme est véritablement attesté et de ne pas considérer que les communautés qui sont administrées par des institutions pérégrines sont nécessairement des castella. En l'état actuel des recherches et faute d'une étude approfondie, aucune des définitions proposées jusqu'ici n'est pleinement satisfaisante. Le sens de castellum comme agglomération sans statut particulier semble néanmoins être celui qui présente le moins de contradictions avec le corpus documentaire. Une telle agglomération peut être dotée d'un territoire, comme le prouvent plusieurs inscriptions ${ }^{66}$, et elle peut être incluse dans le territoire d'une cité ${ }^{67}$. Cette définition présente l'intérêt d'embrasser les différents cas de figure, comme les castella de la Confédération cirtéenne, les castella gérés par des magistrats pérégrins du territoire de Sicca Veneria ${ }^{68}$, le castellum Victoriae sur le territoire d'Igilgili ${ }^{69}$, mais aussi les castella maurétaniens regroupant des colons des domaines impériaux. Cette solution permet également de faire l'économie de l'hypothèse de la polysémie du mot au sein des provinces africaines ${ }^{70}$. Cette définition n'est par ailleurs pas très éloignée de celle proposée par Isidore dans les Étymologies $(\mathrm{XV}, \mathrm{II}, 11)$ : uici et castella et pagi hi sunt qui nulla dignitate ciuitatis ornantur, sed uulgari hominum conuentu incoluntur et propter paruitatem sui maioribus ciuitatibus adtribuuntur.

\section{BIBLIOGRAPHIE}

\section{Abréviations}

- AAA = GSELL Stéphane, Atlas archéologique de l'Algérie, Alger, Jourdan, 1911.

- $A E=$ L'Année épigraphique.

- CIL = Corpus Inscriptionum Latinarum, vol. VIII : Inscriptiones Africae Latinae. Collegit G. Wilmanns, edidit Th. Mommsen, Berlin, De Gruyter, 1881.

- IAM = LABORY Nadine, Inscriptions antiques du Maroc, 2 : Inscriptions latines, Supplément, Paris, CNRS, 2003.

- ILAlg = Inscriptions latines de l'Algérie, t. II, $1:$ Inscriptions de la confédération cirtéenne, de Cuicul et de la tribu des Suburbures, recueillies par St. Gsell, préparées par E. Albertini et J. Zeiller, publiées par H.-G. Pflaum, sous la dir. de L. Leschi, Paris, 1957 ; II, 2, recueillies par St. Gsell, publiées par H.-G. Pflaum, Alger, 1976 ; II, 3, recueillies et éditées par H.-G. Pflaum, publiées par X. Dupuis, Paris, 2003.

- ILAfr = CAGNAT René, MERLIN Alfred \& CHATELAIN Louis, Inscriptions latines d'Afrique (Tripolitaine, Tunisie, Maroc), Paris, Leroux, 1923.

- ILPBardo = BENZINA BEN ABDALLAH Zeïneb, Catalogue des inscriptions latines païennes du Musée du Bardo, Collection de l'École française de Rome, 92, Rome, 1986.

- FIRA = Fontes Iuris Romani Antejustiniani, ediderunt S. Riccobono, J. Baviera, C. Ferrini, J. Furlani, V. Arangio-Ruiz. Editio altera. Leges, Auctores, Liber Syro-Romanus, Negotia. Pars Altera: Auctores. Edidit J. Baviera. Libri Syro-Romani interpretationem a C. Ferrini confectam iterum edidit J. Furlani, Barbera, Florence, 1940. 


\section{Références}

AOUNALLAH Samir, « Le pagus en Afrique romaine », L'Africa romana, 18, 2010a, p. 1615-1630.

AOUNALLAH Samir, Pagus, castellum et civitas. Étude d'épigraphie et d'histoire sur le village et la cité en Afrique romaine, Pessac, Ausonius, 2010b.

BARONI Anne-Florence, « La praefectura iure dicundo des colonies contribuées de la Confédération cirtéenne ", dans S. Evangelisti \& C. Ricci (éd.), Le forme municipali in Italia e nelle province occidentali tra i secoli I a.C. e III d.C. (Atti della « XXI Rencontre franco-italienne sur l'épigraphie du monde romain », Campobasso, 24-26 settembre 2015), Bari, Edipuglia, 2017, p. 245-253.

BELKAHIA Thouraya, « La libertas de Thala ou l'accès de la cité au rang de colonie », dans S. Mokni \& M. Sebaï (éd.), Institutions municipales en Afrique proconsulaire, Sfax, CAEU - Med Ali Éditions, Laboratoire d'études et de recherches interdisciplinaires et comparées (LERIC), Faculté des Lettres et Sciences humaines de Sfax, 2017.

BEN ABDALLAH Zeineb, « Nouveaux documents épigraphiques d'Ammaedara. Contribution à l'histoire religieuse et municipale sous le Haut-Empire », dans F. Baratte, F. Béjaoui \& Z. Ben Abdallah (éd.), Recherches archéologiques à Haïdra. Miscellanea 2, Rome, École française de Rome, 1999, p. 1-31.

BEN ABDALLAH Zeineb, « À propos d'un nouveau qualificatif du dieu Mars dans une inscription découverte à Thala ", Africa, 18, 2000, p. 9-13.

BERTRANDY François, « Thibilis (Announa), de Juba I au triumvir M. Aemilius Lepidus. Les premières étapes de la romanisation d'une cité numide (46-36 av. J.-C.) », Karthago, 19, 1977, p. 87-106.

BESCHAOUCH Azzedine, « Le territoire de Sicca Veneria (El-Kef), nouvelle Cirta, en Numidie proconsulaire (Tunisie) », CRAI, 125, 1981, p. 105-122.

BESCHAOUCH Azzedine, «Colonia Mariana “Augusta” Alexandriana Uchitanorum Maiorum. Trois siècles et demi d'histoire municipale en abrégé », dans M. Khanoussi \& A. Mastino (éd.), Uchi Maius, vol. 1 : Scavi e ricerche epigrafiche in Tunisia, Sassari, Editrice democratica sarda, 1997, p. 97-104.

CAPOGROSSI COLOGNESI Luigi, Persistenza e innovazione nelle strutture territoriali dell'Italia romana. L'ambiguità di una interpretazione storiografica e dei suoi modelli, Naples, Jovene, 2002.

CARCOPINO Jérôme, « Les « Castella » de la plaine de Sétif d'après une inscription latine récemment découverte ", Revue africaine, 1918, p. 5-22.

CRAWFORD Michael Hewson (éd.), Roman Statutes, vol. I, Londres, University of London, 1996.

CHRISTOL Michel, « Les troubles en Maurétanie césarienne sous le gouvernement de T(itus) Licinius Hiéroclès ", dans Y. Le Bohec (éd.), L'Afrique, la Gaule, la religion à l'époque romaine. Mélanges à la mémoire de Marcel Le Glay, Bruxelles, Latomus, 1994, p. 254-266 ; repris dans Id., Regards sur l'Afrique, Paris, Errance, 2005, p. 40-45.

CHRISTOL Michel, « À propos des Anicii : le III siècle », MEFRA, 98, 1986, p. 141-164.

CHRISTOL Michel, « Uchi Maius et Carthage, I : C(aius) Marius C(ai) f(ilius) Arn(ensi tribu) Extricatus », AntAfr, 40-41, 2004, p. 85-98.

CHRISTOL Michel, «La liberté recouvrée d'Uchi Maius et les sources de Pline l'Ancien », dans Regards sur l'Afrique, Paris, Errance, 2005, p. 159-166. 
CHRISTOL Michel \& MOKNI Salem, « L'Histoire naturelle de Pline l'Ancien et la pertica de Carthage ", dans S. Mokni \& M. Sebaï (éd.), Institutions municipales en Afrique proconsulaire, Sfax, CAEU - Med Ali Éditions, Laboratoire d'études et de recherches interdisciplinaires et comparées (LERIC), Faculté des Lettres et Sciences humaines de Sfax, 2017, p. 11-28.

DESANGES Jehan, Pline l'Ancien - Histoire naturelle, Livre V, 1-46, $1^{\text {re }}$ partie, texte établi, traduit et commenté par J. Desanges, Paris, Les Belles Lettres, 1980.

DONDIN-PAYRE Monique, «Compte rendu de S. Aounallah - Pagus, castellum, et Ciuitas. Études d'épigraphie et d'histoire sur le village et la cité dans l'Afrique romaine, Bordeaux, Ausonius, 2010 ", L'Antiquité classique, 81, 2012, p. 517-519.

DUPUIS Xavier, «Les Inscriptions latines de l'Algérie », dans S. Demougin, X. Loriot, P. Cosme \& S. Lefebvre (éd.), H.-G. Pflaum, un historien $d u x^{e}$ siècle (Actes du colloque international, Paris, les 21, 22 et 23 octobre 2004), Genève, Droz, 2006, p. 39-47.

ERNOUT Alfred \& MEILLET Alfred, Dictionnaire étymologique de la langue latine. Histoire des mots, Paris, Klincksieck, 2001 (retirage de la 4 e édition augmentée d'additions et de corrections par Jacques André).

FEVRIER Paul-Albert, «Inscriptions inédites relatives aux domaines de la région de Sétif », dans R. Chevallier (éd.), Mélanges d'archéologie et d'histoire offerts à André Piganiol, t. I, Paris, SEVPEN, 1966, p. 217-232.

GASCOU Jacques, «L'emploi du terme respublica dans l'épigraphie latine d'Afrique », MEFRA, 91, 1979, p. 383-398.

GASCOU Jacques, « Les magistratures de la Confédération cirtéenne », BCTH, n. s. 17, fasc. B, 1981, p. 323-335.

GASCOU Jacques, « La politique municipale de Rome en Afrique. II : Après la mort de Septime Sévère ", ANRW, II (10/2), 1982, p. 230-320.

GASCOU Jacques, «Pagus et castellum dans la Confédération cirtéenne », AntAfr, 19, 1983, p. 175-207.

GSELL Stéphane \& JOLY Charles Albert, Khamissa, Mdaourouch, Announa, vol. III : Announa, Alger/ Paris, Gouvernement général de l'Algérie, 1918.

GSELL Stéphane, Histoire ancienne de l'Afrique du Nord, t. V : Les royaumes indigènes. Organisation sociale, politique et économique, Paris, Hachette, 1927.

GUILLAUMIN Jean-Yves \& MONAT Pierre, Isidore de Séville - Étymologies, Livre 15 : Les constructions et les terres, texte établi, traduit et commenté par J.-Y. Guillaumin, avec la collaboration de P. Monat, Paris, Les Belles Lettres, 2016.

HEURGON Jacques, « Les origines campaniennes de la Confédération cirtéenne », Libyca, 5, 1957, p. 7-24.

HUMBERT Michel, « Municeps et municipium : définition et histoire », dans L. Capogrossi Colognesi \& E. Gabba (éd.), Gli Statuti municipali, Pavie, Iuss Press, 2006, p. 3-29.

JULIEN Yvette, Aulu-Gelle - Les nuits attiques. Tome IV, Livres XVI-XX, texte établi et traduit par Y. Julien, Paris, Les Belles Lettres, 1998.

KAABIA Ridha, « La grotte Ez Zemma un lieu de culte extra-urbain marqueur des propriétés des Phuenses en Cirtéenne (Numidie) », dans T. Belkahia, L. Ben Abid \& M. Gharbi (éd.), Identités et territoires dans le Maghreb antique, Tunis, Université de Tunis, 2016, p. 163-188. 
KALLALA Nabil, «Civitas Titulitana : Mahjouba (région du Kef, au nord-ouest de la Tunisie) », Africa, 17, 1999, p. 87-95.

KHANOUSSI Mustapha \& MAURIN Louis, Dougga, fragments d'histoire. Choix d'inscriptions latines éditées, traduites et commentées ( $I^{e r}-I V^{e}$ siècles), Bordeaux/Tunis, Ausonius/Institut national du Patrimoine, 2000.

LE BOHEC Yann, « Dimmidi. (Demmed - Castellum) », dans G. Camps (éd.), Encyclopédie berbère, vol. 15, Aix-en-Provence, Édisud, 1995, p. 2345-2349.

LEPELLEY Claude, Les cités de l'Afrique romaine au Bas-Empire, t. I, Paris, Études augustiniennes, 1979.

LEPELLEY Claude, Les cités de l'Afrique romaine au Bas-Empire, t. II, Paris, Études augustiniennes, 1981a.

LEPELLEY Claude, « Notes sur sept inscriptions africaines du Bas-Empire », ZPE, 43, 1981b, p. $185-193$.

LEVEAU Philippe, «Introduction : les incertitudes du terme villa et la question du vicus en Gaule narbonnaise », RAN, 35 (1), 2002, p. 5-26.

LEVEAU Philippe, «Vicus, “agglomération secondaire”. Des mots différents pour une même entité ? ", dans A. Ferdière \& C. Cribellier (éd.), Agglomérations secondaires antiques en région Centre, $42^{\mathrm{e}}$ Supplément à la Revue archéologique du Centre de la France, 2012, p. 165-175.

MASTINO Attilio, «Un decurione dell'ala III Asturum praepositus castelli Tamudensis, in una nuova dedica a Giove nel dies natalis di Settimio Severo », MEFRA, 102 (1), 1990, p. 247-270.

MOKNI Salem, « Les premiers temps de la Carthage romaine » et la titulature de la colonie », CCG, 19, 2008, p. 53-76.

NADDARI Lotfi, « Entre coloni et Musulamii : une opération de délimitation des terres sous Trajan dans la vallée de l'Oued Sarrat ", dans F. Béjaoui (éd.), Actes du $5^{e}$ colloque international sur l'histoire des steppes tunisiennes (Sbeïtla, 5-7 mai 2006), Tunis, Institut national du Patrimoine, 2008, p. 157-183.

PFLAUM Hans-Georg, « La romanisation de l'ancien territoire de la Carthage punique à la lumière des découvertes épigraphiques récentes ", AntAfr, 4, 1970, p. 75-118; repris dans L'Afrique romaine. Scripta varia I, Paris, L'Harmattan, 1978.

PICARD Gilbert-Charles, Castellum Dimmidi, Alger/Paris, De Boccard, 1947.

REBUFFAT René, « Castellum », dans G. Camps (éd.), Encyclopédie berbère, vol. 12, Aix-en-Provence, Édisud, 1993, p. 1822-1833.

SISANI Simone, In pagis forisque et conciliabulis: le strutture amministrative dei distretti rurali in Italia tra la media repubblica e l'età municipale, Rome, Accademia nazionale dei Lincei, 2011.

TARPIN Michel, Vici et pagi dans l'Occident romain, Rome, École française de Rome, 2002.

\section{ANNEXES}

PIR ${ }^{2}=$ Prosopographia Imperii romani saec. I-II-III. Editio altera, Berlin/Leipzig, puis Berlin/ Boston, W. de Gruyter, 1933-2015. 


\section{NOTES}

1. Ce travail s'est nourri des discussions avec Clément Chillet, Marie-Claire Ferriès et Elsa Rocca, que je remercie tous trois pour leurs conseils amicaux.

2. Par exemple Lex de Gallia Cisalpina, XXI, 1-4 dans Crawford (1996, 461-477, $\left.\mathrm{n}^{\circ} 28\right)$ : [...] o(ppido) $m$ (unicipio) c(olonia) $p$ (raefectura) / $f($ oro $) u($ eico) $c$ (onciliabulo) $c$ (astello) t(erritorio)ue, quae sunt eruntue in Gallia Cisalpeina [...]. Également Paulus, Sententiae, 4, 6,2 (FIRA, $\mathrm{II}^{2}$, p. 376): Testamenta in municipiis coloniis oppidis praefectura uico castello conciliabulo facta in foro uel basilica praesentibus testibus uel honestis uiris inter horam secundam et decimam diei aperiri recitarique debebunt.

3. Cette difficulté se reflète dans la variété des traductions du terme en français, par exemple «bourgades » ou «bourgs » par Rebuffat $(1993,1822)$, «bourgade », « village », « hameau » ou « petit centre urbain » par Bertrandy (1977, 88-90), «village» dans Aounallah (2010b) - sur ce mot, voir la critique de Dondin-Payre (2012) -, ou «agglomération secondaire » chez Christol $(2005,164)$.

4. Rebuffat (1993).

5. Voir par exemple les réflexions de Leveau (2012, en part. 167). Des travaux collectifs sur le castellum sont par ailleurs en cours dans le cadre des programmes Habitat groupé en Méditerranée occidentale (Casa de Velázquez), dirigé par A.-F. Baroni, E. Rocca et R. González Villaescusa et Communautés, statuts, territoires (Université Grenoble Alpes) dirigé par A.-F. Baroni, C. Chillet, M.C. Ferriès et $\mathrm{E}$. Rocca.

6. Ces deux derniers aspects correspondent ainsi aux deux définitions du castellum proposées par Aounallah (2010b). Contrairement à ce que pouvait laisser présager son titre, Pagus, castellum et ciuitas, l'ouvrage est principalement consacré à la documentation relative au pagus en Afrique proconsulaire; il s'appuie sur la bibliographie antérieure pour retenir deux sens de castellum: « chef-lieu du [pagus] » ou « agglomération pérégrine dépourvue d'autonomie ».

7. Par exemple Tarpin $(2002,21)$, Dondin-Payre $(2012,519)$.

8. Cf. Ernout \& Meillet $(2001,104)$.

9. Trad. Guillaumin \& Monat (2016).

10. Rebuffat (1993, 1822). Sur castellum Dimmidi, voir Picard (1947) et Le Bohec (1995). Sur le castellum Tamu[den]sis, voir Mastino (1990), d'où AE, 1991, $1743 ;$ IAM, 2² 848.

11. Voir les sources réunies par Gsell $(1927,240)$, repris par Rebuffat $(1993,1822)$; également Bertrandy $(1977,88-90)$ : liste des occurrences de castellum, avec les deux sens de «fortin » ou de petit centre urbain, dans le Bellum Africum.

12. Gascou $(1979,393$, n. 46$)$ : «Le terme castellum ne constitue pas une définition juridique très précise, et implique seulement l'existence d'une agglomération fortifiée. » Rebuffat $(1993,1822)$ : "Ajoutons que dans le castellum de la Moulouya (Jug. 92) perché sur un sommet presque inaccessible, il y a des femmes et des enfants, ce qui prouve que ce n'est pas un "fortin", comme on le croit si souvent, mais un hameau fortifié.». Leveau $(2002,10)$, à propos d'Isidore, Etymologiae, XV, 2, 11 sq.: «[Le castellum] partage avec le uicus la situation de dépendance juridique envers la cité, mais il s'en différencie par la possession d'un rempart. » Au début de la description de l'Afrique, Pline, Histoire naturelle, V,1 oppose les oppida qui semblent être de véritables villes aux castella que Desanges (1980) traduit par «fortins": Populorum eius oppidorumque nomina uel maxime sunt ineffabilia praeterquam ipsorum linguis; et alias castella ferme inhabitant. ["Les noms de ses peuples et de ses villes sont tout particulièrement imprononçables pour d'autres bouches que celles des indigènes, et du reste ils habitent en général de simples fortins. »]

13. Desanges $(1980,79)$.

14. Trad. personnelle.

15. Par exemple en Maurétanie césarienne, en 128, CIL, VIII, 8369: Termini positi inter / Igilgilitanos, in / quorum finibus kas/tellum Victoriae / positum est, et Zimiz(es), / ut sciant Zimizes / non 
plus in usum / se haber(e), ex aucto/ritate M(arci) Vetti La/tronis, proc(uratoris) Aug(usti), / qua in circuitu / a muro kast(elli) p(assus) / D; (anno) pr(ouinciae) LXXXIX, Tor/quato et Libone co(n)s(ulibus). Sous Gordien III, sont agrandis les murs de plusieurs castella de la plaine de Sétif - le castellum Vanarzanense (AE, 1903, 94), le castellum Lemellefense (CIL, VIII, 20602) et le castellum Thib(---) (CIL, VIII, 20487) ; cf. Rebuffat (1993). Comme la construction des murs du castellum Thib(---) date du règne de Sévère Alexandre (cf. infra), il n'est pas impossible que les deux autres castella aient été pourvus d'une enceinte à la même époque.

16. Carcopino (1918), d'où $A E, 1917 / 1918,68$ (en 227) : Infatigabili indulgen(t)ia / domini $n$ (ostri) Seueri / Alexandri Pii Felicis Aug(usti), / auctis uiribus et moeni/bus suis, castellan(i) Cito/factenses muros extrux/erunt, curante [-] Licin(i)o Hie/roclete, procuratore Aug(usti), / praeside prouinciae, a(nno) p(rouinciae) CLXXXVIII. Voir Christol (1994).

17. Février (1966, 220-225), d'où $A E, 1966,593$ : Infatigabili indulgentia/dom(ini) n(ostri) Seueri [[Alexandri]] / Pii Felicis Aug(usti), auctis uirib/us et moenibus suis, castellani / Perdicenses muros extruxe/runt, curante Licinio Hie/roclete, procuratore / Aug(usti), praeside prouinciae, / a(nno) p(rouinciae) CLXXXXVIII. Voir Christol (1994).

18. Février (1966, 225), d'où $A E, 1966,594$ : [Infatigabili indulgentia dom(ini) n(ostri) Seueri Alexandri Pii Felicis Aug(usti), aucti]s uirib[us et moenibus suis] castellani [--- m] uros extru[xerunt, curante Lici]nio Hi[eroclete, procu]ra/tore [Augusti, praeside pr]ouinciae, [a(nno) p(rouinciae) CLXXXVIII].

19. CIL, VIII, 8701 : Imp(erator) Caesar M(arcus) / Aurelius Seuerus / [[Alexander I]]nuictus / Pius Felix Aug(ustus), muros / kastelli Diane(n)sis ex/trux[i]t per colonos eiusde/m kastelli, / p(rouinciae) CLXXXXV. 20. CIL, VIII, 20486: [Infatig]abili indul[gentia] / [domini] $n$ (ostri) Seueri [[Alexandri]] / [Pii Felicis] Aug(usti), auctis uiri/[bus et moen]ibus suis kast/[ellani Thib(---) ---] mu/[ros ---].

21. Carcopino (1918, 10). Voir aussi Rebuffat (1993, 1823-1824).

22. CIL, VIII, 8777 : Pro salute et incolu/mitate domini nostri Imp(eratoris) Ca[es(aris)] / M(arci) Antoni Gordiani Inuicti Pii Fe/licis Aug(usti) totiusque domus / diuinae eius, murus constitu/tus a solo a colonis eius cas/telli Cellensis, dicatissi/me deuoti numini eius, / fecerunt, a(nno) p(rouinciae) CLXXXXXIIII.

23. Trad. Guillaumin \& Monat (2016).

24. Trad. J. Gascou, citée par Leveau $(2002,10)$; voir également Leveau $(2012,171)$.

25. C'est par exemple le cas pour les castella de la Confédération cirtéenne dans le CIL, VIII, p.586: (Phua) pagus castellumue; indices, p. 279: pagus et castellum promiscue. Douze agglomérations appartenant au territoire de Cirta sont des castella, mais c'est seulement sur trois sites, à Celtianis, à Sigus et au castellum Phuensium, que sont attestés à la fois un pagus et un castellum (ILAlg, II, 1, 2096 ; ILAlg, II, 2, 2240 ; ILAlg, II, 2, 6519 ; ILAlg, II, 2, 6521 ; ILAlg, II, 2, 4372 ; ILAlg, II, 2, 6486 ; ILAlg, II, 3, 8956). Avant les études sur le pagus de ces dernières décennies, persistait une ambiguïté sur la signification du terme, qui pouvait être compris à la fois comme district territorial mais aussi comme agglomération ; ainsi Lepelley $(1979,132)$ : « Les bourgades et villages dépendant des cités étaient appelés uici, pagi ou castella. L'institution du pagus était complexe sous le Haut-Empire ; elle désignait fondamentalement un territoire rural. [...] Des pagi dépendaient aussi de Cirta et le terme semble avoir servi à désigner des agglomérations. »

26. Pour ce travail, on laissera de côté le présupposé de «l'exception cirtéenne » et les castella cirtéens ne seront pas traités à part. La Confédération cirtéenne, expression moderne qui désigne ce que les inscriptions nomment res publica IIII coloniarum Cirtensium, représente sous le HautEmpire «un extraordinaire hapax administratif», selon le mot communément emprunté à J. Heurgon $(1957,8)$ : la vaste pertica de Cirta comprend plusieurs agglomérations en dehors du chef-lieu de cité, dont trois Chullu, Rusicade et Mileu, portent le titre de colonie sur les inscriptions des $\mathrm{II}^{\mathrm{e}}$ et $\mathrm{III}^{\mathrm{e}}$ siècles, alors qu'il s'agit de communautés contribuées à Cirta (ILAlg, II, 3, 8559). Parmi les autres agglomérations, plus modestes, de ce territoire, certaines sont attestées comme pagus et/ou castellum (voir note précédente). La res publica IIII coloniarum cesse d'exister au milieu du III siècle, entre le règne de Trébonien Galle et Volusien, en 251-253 (ILAlg, II, 1, 625 et 3596 ; cf. Christol, 1986, 153), et celui de Claude le Gothique : en 270, un milliaire est élevé par le municipe 
de Thibilis (Lepelley, 1981a, 383, 478 et n. $17 ; 1981$ b, 191-193; d'où AE, 1982, 953), autrefois pagus de Cirta (infra n. 31). La dissolution de la Confédération cirtéenne intervient probablement sous Gallien, sous le règne duquel sont connues les dernières promotions municipales en Afrique (Gascou, 1982, 317). Mileu (ILAlg, II, 3, 8559), comme probablement les deux autres anciennes colonies contribuées, devient alors une véritable colonie. La promotion au rang de municipe de Thibilis (attesté comme pagus de Cirta, mais non comme castellum) indique que d'autres anciennes dépendances cirtéennes ont pu être également détachées du territoire de Cirta au milieu du III ${ }^{\mathrm{e}}$ siècle et devenir de véritables cités. Malheureusement, aucune source ne nous renseigne de façon claire sur le statut des castella de la Confédération cirtéenne à partir de la seconde moitié du III ${ }^{\mathrm{e}}$ siècle. Cette lacune est d'autant plus regrettable que, grâce à la riche documentation épigraphique du nord de la Numidie, les castella cirtéens sont sans doute les mieux connus des provinces africaines pour le $\mathrm{II}^{\mathrm{e}}$ siècle et la première moitié $\mathrm{du} \mathrm{III}^{\mathrm{e}}$ siècle. On suit en particulier leur évolution institutionnelle, d'abord avec un conseil décurions et un trésor public au début du II siècle, puis une res publica qui apparaît dans les dépendances de Cirta (sauf dans les colonies contribuées) à la fin du $\mathrm{II}^{\mathrm{e}}$ siècle ou au début du III ${ }^{\mathrm{e}}$ siècle, selon les cas : cf. Gascou (1983). Cette richesse documentaire et l'existence des quatre colonies cirtéennes ont souvent conduit les historiens à considérer Cirta et ses dépendances comme des exceptions et empêché la comparaison avec les autres cités. Pourtant l'«hapax» cirtéen semble être limité aux trois colonies contribuées et à leur administration par des triumvirs. Cette dernière a par ailleurs été en partie expliquée par J. Gascou $(1981,325-326)$ : l'ajout pragmatique d'un troisième magistrat au collège des duumvirs habituel au cours $d u{ }^{\text {er }}$ siècle a probablement pour but de gérer l'immense territoire colonial, dans un contexte de prospérité économique et de développement des agglomérations de Chullu, Mileu et Rusicade. Pour le reste, le fonctionnement des institutions cirtéennes ne paraît pas être aussi singulier qu'on le dit habituellement, comme le montre l'exemple de la praefectura iure dicundo: voir Baroni (2017). Au contraire, l'organisation du territoire cirtéen ressemble largement à celle de la pertica de Carthage. La création de la colonie de Cirta par Octavien, avant 26 av. J.-C. (ILAlg, II, 2, 4226), a lieu très vraisemblablement en même temps que la refondation de Carthage en 28 av. J.-C. (Mokni, 2008): dans les deux cas, les autorités romaines ont procédé suivant un schéma attesté dans le reste de l'Empire, à savoir l'organisation des territoires conquis par grands ensembles; de petites communautés, considérées comme inaptes à s'administrer elles-mêmes, sont ainsi placées dans la pertica et sous la dépendance d'une colonie. Entre le $\mathrm{I}^{\mathrm{er}}$ siècle et le $\mathrm{III}^{\mathrm{e}}$ siècle, ces communautés obtiennent progressivement des institutions qui leur confèrent une autonomie croissante pour la gestion des affaires locales et peuvent parfois prétendre au III ${ }^{\mathrm{e}}$ siècle (comme Thibilis) à la promotion civique. Le processus est bien étudié pour les dépendances de Carthage. Or les étapes de cette évolution institutionnelle, mises notamment en évidence pour le pagus d'Uchi Maius - p. ex. Christol (2004), Christol (2005, 161), également Christol \& Mokni (2017) - sont identiques à celles dont ont bénéficié, avec cependant quelques années de retard, les dépendances de Cirta. Pour l'étude des communautés de la pertica, l'organisation cirtéenne est donc comparables à celle d'autres cités africaines, plutôt que comme une exception dont la documentation devrait nécessairement être traitée à part.

27. AAA, f. 17, $\mathrm{n}^{\circ} 126$, p. 12. Gsell \& Joly $(1918,22)$ : «Ce qui, en droit, distinguait surtout les pagi des cités, c'était que les centres de population des pagi n'avaient aucune existence officielle. Dans la cité, la ville était l'organe essentiel, dont la campagne n'était qu'une annexe ; dans le pagus, le district seul comptait au point de vue administratif. Cependant, le bourg, le castellum, centre économique et religieux du canton, lieu de réunion du conseil, résidence des familles les plus aisées, avait, en fait, une importance telle que cette conception juridique du pagus ne se maintint pas intacte. Sur des inscriptions de la région de Cirta, l'expression magister castelli se substitue quelquefois à celle de magister pagi. Le castellum joue, dans la pseudo-commune, le rôle de l'urbs dans une commune véritable. » 
28. Notamment Capogrossi Colognesi (2002), Tarpin (2002), Sisani (2011).

29. Aounallah (2010b, 16, 82). Voir également Aounallah (2010a, 1630).

30. Castellum Elefant(...) (AAA, f. 17, 93) ; castellum Mastarense (AAA, f. 17, 94) ; castellum Phuensium (AAA, f. 17, 102); castellum Arsacalitanum (AAA, f. 17, 111).

31. Le pagus est attesté grâce à la présence d'un magister pagi dans cinq seulement des agglomérations dépendantes de Cirta: Celtianis (ILAlg, II, 1, 2096), Sigus (ILAlg, II, 2, 6519, 6521, 4653), Thibilis (ILAlg, II, 2, 4630, 4641, 4653), le castellum Phuensium (nombreuses dédicaces dans la grotte Ez Zemma du Chettaba, p. ex., ILAlg, II, 3, 8910-8912), et vraisemblablement El Hanacher, près de Cirta (ILAlg, II, 1, 2047).

32. AAA, f. 8, 173.

33. ILAlg, II, 1, 3445 : [---] Castela / [---]alla / [C]ald(is) [---].

34. Supra n. 27.

35. Par exemple Tarpin (2002), Sisani $(2011,608)$.

36. Beschaouch $(1997,102)$; Desanges $(1980,79)$ : « agglomération indigène fortifiée par l'art ou par l'assise ".

37. Rebuffat $(1993,1822-1823,1825)$.

38. CIL, VIII, 8426.

39. Lepelley $(1979,132)$ : «On peut affirmer que les bourgades et petites villes qui ne possédaient pas de statut municipal de plein exercice portaient le titre de castellum "; et n. 55bis : "Ceci ressort d'un canon du concile de Carthage de 403 : les évêques décident de faire convoquer dans chaque lieu l'évêque donatiste par les autorités, les magistrats (c'est-à-dire les duumvirs) dans les cités, les seniores dans les autres lieux; or, les seniores étaient responsables de l'administration locale dans les castella. »

40. Beschaouch (1981, en part. 115-116 ; 121) : les castella du territoire de Sicca et de Cirta seraient «des agglomérations urbaines numides, de statut pérégrin» (Beschaouch, 1997, 102); repris par exemple par Kallala (1999), Naddari (2008, 170), Aounallah $(2010 b, 16)$. En outre, pour Aounallah $(2010 \mathrm{a}, 1617,1624)$, le terme correspondrait à un statut inférieur à celui de ciuitas. Ce dernier point semble difficile à admettre car si Sutunurca est un castellum - d'après la lecture ILAfr, 301 dans Beschaouch (1997, 102) - avant de devenir ciuitas (CIL, VIII, 24004), d'autres communautés, au contraire, sont d'abord connues comme ciuitates puis comme castella. Ainsi, Biracsaccar, en Proconsulaire, est une ciuitas administrée par des suffètes et un conseil des décurions sous Antonin le Pieux (CIL, VIII, 23876) puis un castellum en 374 (CIL, VIII, 23849; ILPBardo, 408) : selon Lepelley (1981, 83-84), « le titre de ciuitas fut peut-être abandonné du fait qu'il impliquait un droit public pérégrin qui n'existait plus ». Contra Pflaum (1970, 86-87) ; repris dans Pflaum (1978, 311-312), pour qui la ciuitas devient castellum après la construction de murailles ; pour Aounallah (2010b, 98-99), le castellum serait ici le chef-lieu de la ciuitas.

41. Aounallah (2010b, 40) : "Chiniaua était une agglomération pérégrine, un castellum. » CIL, VIII, 25450 signale un ordo Chiniauensium peregrinorum. Toutefois, Pline, Histoire naturelle, V, 29-30 compte Chiniaua parmi les oppida ciuium Romanorum XV. La précision peregrinorum sur l'inscription CIL, VIII, 25450 indique probablement qu'il existait à Chiniaua un ordo de citoyens romains et un autre de pérégrins.

42. Selon Lepelley $(1981,315)$ repris par Ben Abdallah $(2000,10)$, « en 209-210, la commune est toujours administrée par des seniores, ce qui implique le statut de castellum pérégrin. Vraisemblablement ce castellum était dans la mouvance d'Ammaedara (ILAfr, 195) ». Sur cette communauté pérégrine, voir Ben Abdallah (1999, 52-53) et Belkahia (2017).

43. Kallala $(1999,89):$ « [...] nous avons affaire tout au plus à un castellum, à cause de la mention des seniores. » Le mot castellum n'est, sauf erreur, pas attesté à Tituli. La ciuitas Titulitana apparaît sur une inscription que l'on peut probablement dater du début du $\mathrm{III}^{\mathrm{e}}$ siècle (Kallala, 1999 ; d'où AE, 1999, 1853). Les seniores de Tituli sont connus par l'inscription CIL, VIII, 27828. 
44. Rebuffat $(1993,1824)$ : «Quand on rencontre des seniores, on peut penser que seul le hasard ne nous procure pas la mention effective d'un castellum : voir par exemple ILAfr 195 à Thala. » 45. CIL, VIII, 15721 (en 213) : Imp(eratori) Caes(ari) M(arco) Aurelio / Antonino Pio Aug(usto) / Fel(ici), Part(hico) max(imo), Brit/[t]an(nico) max(imo), German(ico)/ max(imo), pont(ifici) max(imo), trib(unicia) / pot(estate) XVI, imp(eratori) II, co(n)[s(uli)] / IIII, p(atri) [p(atriae), pr]oco(n)s(uli), opti/mo $m[$ aximo]que / principi, seniores / $k$ (astelli) posuer(unt) et ded(erunt).

CIL, VIII, 15722 : Iuliae Aug(ustae) / Piae Felici, / matri Augus/ti et castro/rum et sena/tus et patriae, / seniores kast(elli) / pos(uerunt) et dedic(auerunt).

46. CIL, VIII, 15669 (fin 213-214) : Imp(eratori) Caes(ari) M(arco) Aurelio / Antonino Pio Aug(usto) Fe/ lici, Parth(ico) max(imo), Brit $[t]$ (annico) / max(imo), Ger(manico) max(imo), pont(ifici) max(imo), / trib(unicia) pot(estate) XVII, imp(eratori) III, co(n)s(uli) IIII, / p(atri) p(atriae), proco(n)s(uli), Optimo Maxi/moque principi, diui/L(uci) Septimi Seueri, Arab(ici), / Adiab(enici), Parth(ici) max(imi), Brit(annici) / max(imi) fil(io), diui M(arci) Antonini, / Ger(manici), Sar(matici) nep(oti), diui Antoni/ni Pii pronep(oti), diui Hadri/ani abnep(oti), diui Traiani et / diui Neruae adnep(oti), decur(iones) / Sic(censes) Ucubi morantes et seni/ores k(astelli) Ucubis, aer(e) conl(ato), [p(osuerunt)], / d(ecreto) d(ecurionum).

47. Communication d'Azzedine Beschaouch dans Rebuffat $(1993,1826)$.

48. La dépendance de ces castella envers Sicca est déduite des inscriptions CIL, VIII, 15726, 15669 et 16367.

49. Outre les castella du territoire de Cirta qui présentent à cet égard le dossier le plus fourni, étudié par Gascou(1983), on peut citer les deux magg(istri) q(uin)q(uennales) kastelli d'une communauté anonyme mentionnés par CIL, VIII, 9317, entre Tipasa et Caesarea, en Maurétanie césarienne.

50. Beschaouch (1981, 117-118).

51. Voir supra n. 46.

52. Cf. Khanoussi \& Maurin (2000).

53. ILAlg., II, 13574 et 3612.

54. Aounallah (2010b, 90-91).

55. Ce castellum avait peut-être ses propres édiles (voir l'inscription de l'arc du Nord, ILAlg, II, $1,3608)$.

56. Au castellum Tidditanorum en 136-138 (ILAlg, II, 1, 3605).

57. ILAlg, II, 1, 3563. Q. Lollius Urbicus (PIR ${ }^{2}$ L 327) est patron du castellum Tidditanorum (ILAlg, II, 1, 3605), mais aussi celui de Caldis (ILAlg, II, 1, 3446), d'après des hommages datés de 136-138.

58. On sait par une autre inscription que ce personnage est promu chevalier sous Septime Sévère et Caracalla. CIL, VIII, 6711 ; ILS, 6863a ; ILAlg, II, 1, 3610 : Aproniae Sex(ti) fil(iae) Fi/dae, coniugi / Q(uinti) Sitti Q(uinti) fil(i) Quir(ina) Faus/ti, probati ab / imp(eratoribus) Septimio Seuero / Pio Pertinace Aug(usto) et / M(arco) Aurelio Antonino Aug(usto), / in quinq(ue) decurias allecti a diuo / M(arco) Antonino Pio, flam(inis) perp(etui), / [III]uir(i) quinq(uennalis), IIIuir(i), praef(ecti) i(ure) d(icundo) / [co]l(oniae) Vener(iae) Rusic(ade), praef(ecti) i(ure) d(icundo) / [c]ol(oniae) Sarn(iae) Mil(eu) et praef(ecti) i(ure) d(icundo) / col(oniae) Miner(uiae) Chullu, aedil(is), amici ob merita marit(us) / [e]ius in se, aere conlato, / l(oco) d(ato) d(ecreto) d(ecurionum). Sur les magistratures de la Confédération cirtéenne, voir Gascou (1981).

59. CIL, VIII, 6710 ; ILS, 6863 ; ILAlg, II, 1, 3611 : Q(uinto) Sittio / Q(uinti) fil(io) Quir(ina) / Fausto, IIIuiro, / praef(ecto) i(ure) d(icundo) col(oniae) Ve/neriae Rusicade / et col(oniae) Sarn(iae) Mileu / et col(oniae) Mineruiae / Ch(u)llu, aedili, / municipes, ob / merita eius, / aere conlato, / d(ecreto) d(ecurionum).

60. Aulu-Gelle, Nuits attiques, XVI, 13, 2: Quotus enim fere nostrum est, qui, cum ex colonia populi Romani sit, non et se municipem esse et populares suos municipes esse dicat? ["Car quelle est la proportion de ceux d'entre nous qui, originaires d'une colonie du peuple romain, ne s'en disent pas municipes et n'appellent pas leurs compatriotes municipes? », trad. Julien (1998), modifiée]. 
61. Gascou (1983, 181, n. 44) et Dupuis (2006, 45-46) ; sur le terme municeps, voir Humbert (2006). Munic[ipes] peut être également lu sur une inscription de Sigus, dépendance de Cirta où sont connus un pagus et un castellum (ILAlg, II, 2, 6520).

62. Les décurions sont clairement ceux du castellum dans deux inscriptions du $\mathrm{III}^{\mathrm{e}}$ siècle (ILAlg, II, $1,3604$ et $A E, 1969-1970,693)$.

63. Gascou (1983, 203 sq.).

64. En dernier lieu, Kaabia (2016).

65. Magister pagi : ILAlg, II, 3, 8911, 8918, 8921, 8933, 8958-8959 et peut-être 8960 ; magister pagi associé aux Phuenses : 8928, 8939, 8953, 8957, et probablement 8909 ; magister pagi associé à la res publica Phuensium : 8910, 8912, 8914-8917, 8929-8931, 8936-8937, 8940 ; magister pagi Phuensium : ILAlg, II, 3, 8938, 8942 (associé à la res publica Phuensium), 8964 ; mag(istri) castelli (ou kastelli) Phuensium : ILAlg, II, 3, 8907-8908, 8948, 8955-8956, 8962 ; mag(ister) P(h)uens(ium): ILAlg, II, 3, 8932 ; magister seul : ILAlg, II, 3, 8913, 8951-8952, 8954 ; magister associé aux Phuenses : ILAlg, II, 3, 8934 ; magister associé à la res publica Phuensium: ILAlg, II, 3, 8920, 8923-8927, 8935, 8941, 8943-8944, 8947.

66. Par exemple, les inscriptions $A E, 1907,5$ près de Sétif et $A E, 1985,972(A E, 1993,1782)$ en 202-209, près de Tipasa signalent le bornage du territoire de castella de Maurétanie. À l'époque augustéenne, des terres ont été octroyées à des colons sur le territoire des castella d'Uchi Maius (CIL, VIII, 26274 et AE, 2006, 1691) et de Sutunurca (ILAfr, 301).

67. Christol $(2005,164)$ : «Le terme de castellum désigne un lieu habité dans le territoire d'une cité, autre que le chef-lieu, ou, dirait-on de nos jours une "agglomération secondaire" : l'exemple de la Confédération cirtéenne voisine vient l'indiquer. »

68. Outre les inscriptions citées plus haut, voir la mention d'un IIuir col(oniae) Sic(cae), pr(a)ef(ectus) caste(lli) : CIL, VIII, 15726.

69. CIL, VIII, 8369 (supra n. 15).

70. Voir, par exemple, Kallala $(1999,91)$.

\section{RÉSUMÉS}

Sous l'Empire romain, le terme castellum revient fréquemment dans la documentation des provinces africaines pour désigner une agglomération rurale. La définition précise de ce terme n'est pas clairement établie et les propositions généralement retenues (un bourg fortifié, le «chef-lieu » d'un pagus, ou une agglomération indigène de statut pérégrin) méritent d'être discutées. Un examen attentif des sources montre qu'aucune de ces trois solutions n'est pleinement satisfaisante. Sous réserve d'une étude plus approfondie, une définition simple du castellum comme agglomération secondaire sans statut particulier semble être une solution prudente.

In the written evidence regarding the African provinces during the Roman Empire, the word castellum often refers to a rural town. The precise definition of this word is not clearly established and the different propositions usually adopted (a fortified small town, the administrative centre of a pagus or a peregrine urban community) deserve discussion. A careful examination of the data shows that none of these three solutions is entirely satisfactory. Subject to a more detailed study, a simple definition of castellum as a small town without any specific status seems to be a prudent solution. 
INDEX

Mots-clés : castellum, Afrique, communautés pérégrines, agglomération rurale

Keywords : castellum, Africa, peregrine communities, rural town

\section{AUTEUR}

\section{ANNE-FLORENCE BARON}

Université Paris 1 Panthéon-Sorbonne

anne-florence.baroni@univ-paris.fr 